Cipango Cahiers d'études japonaises

Hors-série | 2008

Autour du Genji monogatari

\title{
Remarques sur une scène bouddhique du Roman du Genji
}

Some Remarks on a Buddhist scene in the Tale of Genji

Jean-Noël Robert

\section{(2) OpenEdition}

1 Journals

Édition électronique

URL : https://journals.openedition.org/cipango/610

DOI : 10.4000/cipango.610

ISSN : 2260-7706

Éditeur

INALCO

\section{Édition imprimée}

Date de publication : 1 janvier 2008

Pagination : 385-401

ISBN : 978-2-85831-170-5

ISSN : 1164-5857

\section{Référence électronique}

Jean-Noël Robert, «Remarques sur une scène bouddhique du Roman du Genji », Cipango [En ligne], Hors-série | 2008, mis en ligne le 04 mars 2012, consulté le 30 juin 2021. URL : http://

journals.openedition.org/cipango/610; DOI : https://doi.org/10.4000/cipango.610

Ce document a été généré automatiquement le 30 juin 2021

\section{(c) (7) (5)}

Cipango est mis à disposition selon les termes de la Licence Creative Commons Attribution - Pas d'Utilisation Commerciale 4.0 International. 


\title{
Remarques sur une scène bouddhique du Roman du Genji
}

Some Remarks on a Buddhist scene in the Tale of Genji

\author{
Jean-Noël Robert
}

1 Qu'un ignorant comme je le suis des choses de la littérature japonaise ose intervenir dans un colloque sur le Roman $d u$ Genji appelle quelques mots d'explication, sinon d'excuse. Comme l'écrit Kūkai au début de sa Convergence des trois doctrines, « mu par le sentiment, l'homme ne peut qu'en emplir son pinceau ", et mon sentiment, à l'occasion d'une lecture cursive du Roman du Genji dans le texte de la collection Iwanami bunko que l'on doit au grand Yamagishi Tokuhei, fut l'étonnement de découvrir disséminées et enchâssées dans le texte des allusions bouddhiques si nombreuses et si discrètes que l'on ne peut qu'être amené à penser que le bouddhisme est bel et bien une dimension dont il est indispensable de tenir compte dans l'exégèse de cette œuvre.

2 Je vais donc tenter de montrer ici, à partir d'un exemple précis, qui n'a pas encore, semble-t-il, été expliqué à cette lumière, que la prise en compte des textes bouddhiques apporte une intelligence plus fine de certains épisodes significatifs ${ }^{1}$.

3 Nous considérerons le chapitre v, Waka-murasaki, ou " Jeune Grémil ${ }^{2}$ ", pour la raison que toute la première partie de ce chapitre abonde en allusions au bouddhisme et à ses textes et que de nombreuses expressions résonnent avec des choses plus familières à qui étudie le bouddhisme. La scène d'ensemble de ce chapitre, s'il faut le résumer, est une «montagne du nord » (kitayama) impossible à situer davantage aux environs de la capitale, où l'on conseille au prince Genji de se rendre, afin de se faire guérir sa «fièvre maligne " (warawa-yami) grâce aux conjurations d'un "pratiquant " (okonaibito) à la réputation de grande efficacité. Le saint homme (hijiri) ayant fait valoir qu'à cause de son grand âge, il ne sortait plus guère de son ermitage (muro) situé loin du monde (yaya fukau iru tokoro narikeri), c'est donc au Prince d'aller à la montagne, ce qu'il fait accompagné de quatre ou cinq intimes (mutsumashiki yotari-itsutari). Nous sommes à la fin du Troisième mois et, si la saison des fleurs est passée à la capitale, les cerisiers de montagne sont en pleine floraison. Le paysage perdu dans les brumes (kasumi) semble fort impressionner le Prince, qui vit d'ordinaire dans un milieu étriqué (tokoroseki onmi 
nite, medurashū obosarekeri). L'ermitage, appelé ici tera, est tout aussi frappant (ito aware nari) pour les visiteurs : le saint homme demeure sur un pic élevé (mine takaku), dans un endroit enchâssé en la roche même (fukaki iwa no naka ni zo). Malgré l'aspect bien piteux de ses vêtements d'excursion (ito itau yatsure-tamaedo), l'ermite reconnaît un illustre visiteur et s'étonne de le voir s'adresser à lui qui, ne songeant plus guère aux choses de ce monde, avait oublié les pratiques magiques (ima ha kono yo no koto wo omoi-tamaeneba, gengata no okonai mo sute-wasurete haberu wo ikade kau owasimasituramu). Il va malgré tout accomplir les rites de guérison.

4 Cependant, de l'ermitage haut perché s'aperçoivent de ci, de là, des demeures monacales (sōbō) et le Prince, qui était sorti pendant les préparatifs du rite, s'interroge sur l'occupant d'un «ensemble de bâtiments bien entretenus, reliés par des passages couverts et entourés de bosquets disposés avec un goût très sûr ${ }^{3}$ » que l'on aperçoit en contrebas. On lui répond que ce sont les quartiers d'un préfet monacal (sōzu) qui s'y est retiré depuis deux ans. Des jeunes filles s'affairant dans le jardin attirent l'attention du Genji, qui descend les épier. C'est ainsi que commence l'épisode de sa rencontre avec Waka-murasaki.

Je n'irai pas plus loin dans le résumé du chapitre et me contenterai de mentionner les claires allusions à des notions bouddhiques faites en des termes suffisamment précis parsemés surtout dans la première partie. Il convient d'abord de relever l'atmosphère un peu irréelle dans laquelle baigne la scène initiale : la brume, les hauteurs du pic, la roche où est encastrée l'ermitage, et le curieux effet de perspective : c'est de ce site hors du monde que la vue plonge sur un temple où vit un moine en confinement entouré de sa parentèle ; on a trois niveaux du monde : la capitale d'où vient le Prince, qui représente la dimension mondaine, le niveau éthéré de l'ermitage auquel il passe directement, et le niveau moyen, mais quand même religieux, qui va l'attirer par ses tentations féminines, sur lesquelles l'auteur insiste avec quelque ironie : Tiens, là-bas des femmes! ou encore la question du Prince : Au fait, et la fille? Et c'est en ce niveau moyen, où il redescend à partir du niveau supérieur, que va s'esquisser une scène de marivaudage sur un arrière-plan bouddhique qui sert de repoussoir.

6 Tout à fait caractéristique est la sentence moqueuse inspirée des écritures que récite le Genji, passant la nuit dans les quartiers du préfet monacal, lorsqu'il entend une dame de compagnie tâtonner dans l'obscurité dans une pièce voisine : hotoke no on-shirube wa kuraki ni irite mo sara ni tagaumajikanaru mono wo "Qui a pour guide l'Éveillé, même lorsqu'il aura pénétré dans les ténèbres, ne saura certes s'égarer ». On ne peut bien sûr manquer d'évoquer le célèbre poème de la grande rivale de Murasaki Shikibu, Izumi Shikibu : kuraki yori / kuraki michi ni zo / irinu beki / haruka ni terase / yama no ha no tsuki, ainsi traduit par Bernard Frank ${ }^{4}:$ «À partir de l'obscur, en un chemin plus obscur encore, il me faut maintenant m'engager. Veille de loin sur moi, Lune de la cime des montagnes. »

7 S'il m'est permis d'insérer ici une brève digression, il est symptomatique du peu de cas que faisaient (j'espère que la phrase est désormais à mettre au passé) du bouddhisme les spécialistes de la littérature japonaise que Yamagishi Tokuhei, dans ses notes du NKBT ${ }^{5}$ renvoie à un endroit erroné du Sūtra du Lotus le fameux passage 從冥入於冥永不 聞佛名 《Ils s'enfoncent d'obscurité en obscurité sans jamais entendre le nom de l'Éveillé6. » Il ne s'agit pas du chapitre II sur les «Expédients salvifiques », mais bel et bien du chapitre VII sur la «Ville fantasmagorique ». Toujours est-il qu'on ne peut manquer de relever la coïncidence : que deux femmes de lettres contemporaines qui ne 
sont pas particulièrement connues pour leur érudition bouddhique fassent allusion au même verset du Lotus en dit long sur l'imprégnation de la culture " lotusienne ", pour reprendre l'adjectif forgé par Bernard Frank, dans la mentalité de l'époque.

Mais il y a, avant cette pseudo-citation, une allusion qui me semble assez claire au Sütra $d u$ Lotus et dont la portée est plus profonde : il nous est dit que l'ancien gouverneur de la Province d'Akashi, bien que retiré du monde, nourrissait de grands desseins pour sa fille $^{7}$; il répétait souvent que, s'il venait à mourir et que celle-ci ne puisse réaliser ces projets ambitieux, que son destin ne soit point celui qu'il avait conçu (moshi ware ni okurete sono kokorozashi togezu, kono omoi-okitsuru sukuse tagawaba), alors, "qu'elle se jette à la mer " (umi ni irine)! Et l'assistance de s'esclaffer en élaborant sur ces paroles l'image qui vient aussitôt à l'esprit des gens de l'époque : "Voilà donc une fillette de grand prix, appelée qu'elle est à devenir l'épouse du roi Dragon des mers! » (kairyūō no kisaki ni naru beki, itsuki-musume nanari). Le mot plaît tant au Prince qu'il le reprend quelques lignes plus bas (je me risque à une traduction presque littérale afin de repérer plus facilement tout à l'heure les reprises de vocabulaire): "Quelle est donc son intention, pour être entré si profondément en ces pensées de fond de mer? Les algues des fonds/ les regards des gens rendront les choses difficiles. » (nani-gokoro arite umi no soko made fukau omoi-iruran? soko no mirume mo mono-mutsukashū).

Ici encore, Yamagishi Tokuhei, s'il comprend naturellement l'allusion bouddhique et parodique de ces échanges, se contente de renvoyer à un sūtra dont le titre même lui semble suffisant pour justifier l'identification. Il s'agit du Sütra du Roi-Dragon des mers, Kairyūō-gyō (tome XV n ${ }^{\circ}$ 598) : il est certain que l'expression Roi-Dragon des Mers se retrouve non seulement dans le titre mais à plusieurs reprises dans le texte même de ce sūtra, qui était souvent récité à Nara au cours des cérémonies destinées à faire tomber la pluie. Il est vrai aussi que le composé ryūgo ou ryū no kisaki, comme le donne ici le texte du roman, se trouve dans ce même sūtra. Il est tout aussi vrai enfin - et pour mon plus grand dam, me permettrai-je d'ajouter - que ni l'une ni l'autre de ces expressions ne se retrouve telle quelle dans le Sūtra du Lotus. Et cependant il m'est impossible, à la lecture de ces lignes, de penser que ce n'était pas plutôt ce dernier sūtra qu'avait en tête l'auteur lorsqu'elle laissait ses personnages jouer avec cette image.

Tout le monde connaît en effet la fameuse scène du chapitre XII, «Don des Dieux » (Daibadatta-hon) du Lotus, où le bodhisattva Mañjuçrî fait l'éloge de la fille du roi des Dragons, ryūōnyo, en concluant qu'elle est capable de réaliser l'Éveil. Deux éminents assistants de l'assemblée, le bodhisattva Amas de Sagesse (Chishaku bosatsu) et le grand et sévère disciple Çâriputra émettent des doutes, d'autant plus qu'il est question que cet Éveil soit réalisé en un instant. Apparaît alors, venue du fond des mers, l'intéressée elle-même, qui va manifester devant tous sa réalisation immédiate de l'Éveil sublime.

11 Il y a un indice textuel qui attire de prime abord l'attention : en décrivant au Bouddha la fille du roi-dragon, Mañjuçrî énumère ses pratiques de surdouée de la Voie bouddhique et déclare, entre autres, qu' « entrée profondément en concentration, elle a eu accès aux enseignements $»^{8}$, le premier vers, lu en japonais fukaku zenjō ni irite, rappelle étrangement le mot d'esprit du Prince fukau omoi-iruran, avec le simple remplacement de zenjō « méditation-concentration », par un terme plus ordinaire mais qui exprime aussi le processus mental, omou. Le passage en sino-japonais du Sūtra tel que je viens de le citer est pratiquement compréhensible à la seule audition et ce n'est pas faire preuve, me semble-t-il, d'érudition particulière que de le connaitre, si l'on 
tient compte de la popularité probable de l'épisode auprès des dames de la cour, qui voyaient une consœur damer le pion au plus savant disciple de Çâkyamuni.

Il se trouve même, d'après moi, un élément qui permet de relier Waka-murasaki à cet épisode lotusien : c'est que la petite dragonne vient d'avoir huit ans 年始八歳 toshi hajimete hassai ; or l'auteur du Genji nous précise que la jeune héroöne du chapitre «a tout juste dix ans " (tō bakari ni ya aramu). Outre que les reines-dragon du Kairyūōogyō ne sont aucunement individualisées et qu'on les compte par millions, on ne peut guère se les imaginer comme des fillettes; si les compagnons du Genji ont parlé de kisaki, c'est en imaginant son avenir en tant que dragon, mais pour ce qui est de la «tranche d'âge » immédiate, c'est bien sûr plutôt de ce personnage célèbre du Lotus qu'il convient de rapprocher la toute jeune Waka-murasaki. Si ce recoupement est permis, ce chapitre, où apparaissent pour la première fois les deux principaux personnages féminins du roman, dame Murasaki et dame Akashi, les présente d'emblée reliées par un lien «lotusien $»^{9}$.

13 Voyons à présent une autre allusion qui renvoie très probablement aussi au Sūtra $d u$ Lotus; il s'agit cette fois de l'échange de poèmes qui a lieu plus tard entre le Prince, le Préfet monacal et l'ermite. Si extraordinaire est la prestance du Genji, ce météore de l'urbanité qui vient illuminer la montagne des anachorètes, que les deux religieux, retirés à des degrés divers du monde ont perçu sa venue comme une épiphanie qui va remuer sous leur pinceau l'imagerie religieuse. Après le poème du Prince, c'est au Préfet monacal de donner sa contribution et, reprenant le thème des fleurs de cerisier évoqué par le premier, il passe à un autre végétal : udonge no / hana machi-etaru / kokochi shite / mi-yama-zakura ni / me koso utsurane " Avec l'impression d'avoir enfin rencontré la fleur d'udumbara, poserais-je encore mes regards sur les fleurs de cerisier?» On sait que cette fleur, udonge ou udonbara en sino-japonais, a une existence mythique en Extrême-Orient; le fondateur du Tendai chinois, Chigi/Zhiyi, explique qu'elle fleurit tous les trois mille ans et signale la venue d'un empereur du monde. En réalité, comme le rappelle Yamagishi Tokuhei dans une note complémentaire (186, p. 432), il s'agit d'un arbre réel, le Ficus glomerata, une sorte de figuier, arbre qui, comme le rappellent les caractères chinois qui forment son nom, 無花果, donne des fruits mais n'a pas de fleurs visibles. Le même érudit donne comme principales sources scripturaires, entre autres, le Sūtra de l'éclat doré 金光明經 et le Sūtra du Lotus. Si la graphie du Lotus est plutôt 優是: 花 que 優量華, la différence ne parait pas pertinente ici, puisque, sans craindre la redondance, le Préfet monacal utilise les deux caractères réunis dans la locution udonge no hana. Le Sūtra du Lotus mentionne l'udumbara sous la forme udonge à propos des hommes exceptionnels; ainsi au chapitre II sur «Les expédients salvifiques»: "Quelqu'un capable d'écouter cette Loi, de telles gens sont difficiles à trouver. Comparés à la fleur du figuier sauvage, qui fait les délices de tous, rare chez les dieux et les hommes, n'apparaissant qu'une fois de temps en temps ${ }^{10} \ldots$.. , pour reprendre un peu plus loin: "Et de telles gens sont très rares, plus encore que la fleur du figuier sauvage.» Le fait que ce soient des hommes dont la rareté est comparée à la fleur donne à penser que c'est bien du Lotus que s'inspire ce poème, en transférant au Prince les qualités de l'auditeur du Sūtra.

14 Si on hésite cependant à affirmer sans phrase que l'on a bel et bien affaire dans ce troisième cas à une évocation du Lotus, les deux premiers exemples devraient nous y conduire; d'autant plus que vient s'ajouter à ces disjecta membra un composant plus englobant de la scène que l'on doit se garder d'oublier : celle-ci est, en fait rythmé par 
la récitation du Sūtra du Lotus telle qu'elle se pratique au cours du rituel dit Concentration (ou samâdhi) du Lotus (hokke-zanmai), pratique de repentance se déroulant dans la salle de ce nom, ainsi que le dit le roman : hokke-sanmai okonau dō no senbō no koe yama-oroshi ni tsukite kikoe-kuru. Il n'est pas sûr que ce soit à l'intention du Prince que le rite, qui peut être long et complexe et pour la description duquel nous renverrons à l'ouvrage maintenant classique de notre érudite amie, Mme Kuo Liying ${ }^{11}$, est ici exécuté ; il se peut qu'il fasse simplement partie des pratiques du Préfet monacal. Il n'empêche malgré tout qu'il constitue comme la musique de fond de cette nuit qui voit le Prince guéri de son mal, mais aux prises avec une nouvelle passion. Ce détail que nous révèle l'auteur sert à mettre en perspective l'ensemble du passage : nous baignons dans le Lotus, même si le Préfet monacal avait auparavant averti Genji qu'il avait à assurer des rites pour le bouddha Amida (Amida-butsu mono shi-tamau dō ni suru koto haberu koro ni namu); il en est à présent revenu pour participer à l'échange des trois poèmes.

15 C'est donc au son de ces psalmodies lotusiennes que je voudrais aborder le dernier poème de cet échange et tenter de montrer qu'il y a une allusion à un épisode bien déterminé de ce sūtra, allusion qui n’a pas été, à ma connaissance, remarquée. Après le Préfet monacal, l'ermite adresse ce poème au Genji : okuyama no / matsu no toboso wo / mare ni akete / mada minu hana no / kao wo miru kana «Au fond des montagnes en mon attente, ayant ouvert, occasion rare, la porte de pin, voici que j'aperçois le visage d'une splendeur jamais vue.» Traduction bien pédestre, mais qui tâche de rendre chaque mot. Après les fleurs de cerisiers du Genji et la fleur d'udumbara du Préfet monacal, on peut s'attendre à ce que l'ermite poursuive l'escalade de l'hyperbole; c'est déjà quelque chose, dira-t-on, que de comparer son visage à une fleur resplendissante, les deux sens du même mot hana, mais il y aurait alors comme une dérobade par comparaison avec le poème précédent, à moins de considérer que l'ermite reprend telle quelle la métaphore du Préfet, ce qui n'est guère satisfaisant. Quelle est en ce cas l'imagerie mise en œuvre par le troisième participant?

16 Le mot-clef de ce poème, si j'ose dire, est «la porte ». Qui plus est, la porte de pin, matsu, dont il est inutile de souligner ici le double sens avec le verbe « attendre ». Il est peu probable que l'ermite attendît le Genji, mais sa venue est prise, nous l'avons dit, par ces religieux plus ou moins solitaires comme une épiphanie, ainsi que l'a déjà souligné la comparaison avec la fleur d'udumbara, une épiphanie au terme d'une attente. Le lecteur conçoit à la première lecture une image assez simple de l'action suggérée par le poème : l'ermite reclus ouvre sa porte et tombe sur le Prince qui passait par là, d'où surprise et émotion. Mais la porte s'ouvre-t-elle dans le bon sens, c'est-à-dire celui que suggère, justement, le bon sens naif : de l'intérieur vers l'extérieur, de l'ermitage vers le monde? Sans préjuger de l'architecture des ermitages de l'époque et en l'absence assez désespérante de portes dans les illustrations du Genji monogatari, on peut penser qu'ils étaient plutôt munis de portes coulissantes, mais on est bien sûr influencé par des constructions bien plus modernes, comme l'ermitage de Bashō, par exemple. Cela ne change rien à la question première : l'ermite ouvre une porte, mais dans quel sens? Peut-on, après la première impression d'ouverture de l'intérieur vers l'extérieur, imaginer l'inverse : il ouvre une porte de l'extérieur pour regarder à l'intérieur ?

17 Il est dans le Sūtra du Lotus une hiérophanie célèbre entre toutes, source d'une iconographie infinie en Chine comme au Japon: c'est celle de l'Ainsi-Venu MaintTrésor (Tahō-nyorai ; sct. Prabhūtaratna) qui apparaît dans son stūpa de matières 
précieuses surgi de la terre pour rester suspendu en plein ciel. On se souvient de la scène décrite dans le chapitre XI « La vision de la pagode de matières précieuses $^{12}$ " (Ken-hōtō-hon) : du stūpa, ou de la pagode, suspendue dans les airs provient une voix louant Çâkyamuni et le Sūtra du Lotus; il est expliqué à l'assistance stupéfaite qu'un bouddha d'un passé et d'un monde fort lointain, du nom de MaintTrésor, « du temps où il pratiquait la voie d'être d'Éveil, avait fait un grand serment: “Si, après que j'aurai obtenu l'état d'Éveillé et que je serai passé en Disparition, il se trouve, dans les terres des dix orients, un endroit où se prêche le Livre de la fleur de la Loi, ma pagode, afin d'écouter ce livre canonique, surgira de terre et apparaîtra devant tous pour porter témoignage et l'approuver par les mots : C'est bien ! " I I est ajouté un peu plus loin une précision : "S'il se trouve quelqu'un pour prêcher le Livre de la fleur de la Loi, sa pagode surgit à chaque fois devant lui, avec son corps intègre (zenshin: double sens de zen : intact et entier) à l'intérieur, qui l'approuve par les mots : “C'est bien, c'est très bien!" » Le porte-parole de l'assemblée déclare alors au Bouddha: "Vénéré du monde, nous souhaiterions voir le corps de cet Éveillé. » Après une grandiose mise en scène consistant en rien moins que la restructuration de notre monde, le bouddha Çâkyamuni s'élève à son tour dans l'espace et, « de l'index droit (以右指 migi no yubi wo motte), ouvrit la porte de la pagode faite des sept matières précieuses (開七寶塔戸 shippōtō no to wo hiraki-tamau). Il en sortit un grand bruit, comme lorsque l'on ouvre les portes d'une ville (開大城門 daijō no mon wo hiraku) en les déverrouillant, et aussitôt toute l'assemblée aperçut l'Ainsi-Venu Maint-Trésor assis sur un trône léonin dans la pagode précieuse, le corps intègre, non dispersé, comme entré en concentration... » Après quoi Maint-Trésor invite Çâkyamuni à prendre place près de lui dans la pagode, et tout le reste de l'assemblée monte à son tour dans les airs. Cette scène a été bien souvent représentée, et il me suffira de renvoyer aux pages qu'a écrites à ce sujet Bernard Frank ${ }^{13}$.

$18 \mathrm{Lu}$ à la lumière de ce passage, le poème prend alors une autre dimension, franchit un nouveau degré dans l'hyperbole élogieuse: si l'ermite a bien ouvert la porte de son ermitage, c'est en répétant à son niveau le geste que fit autrefois çâkyamuni pour découvrir le corps splendide de Maint-Trésor enchâssé en sa pagode. Il ouvre la porte de sa cabane, qui devient, inversée, la porte de la pagode. La splendeur du visage, hana, rappelle la «fleur de la Loi », hokke / nori no hana, qui est la raison d'être de l'apparition du stūpa, superposée au visage qui évoque le corps intègre de Maint-Trésor. Dans le Sūtra, l'udumbara, si rare, servait de comparaison pour ceux qui accueillaient dignement le Lotus ; ici, la comparaison porte sur un bouddha. Relevons en passant que le mare ni " rare occasion » du troisième poème reprend l'adjectif keu, " rare », épithète par deux fois de l'udumbara dans le passage du Sūtra mentionné plus haut; il y a continuité avec le deuxième poème et dépassement. De plus, le fait qu'il était précisé au début du chapitre que l'ermite résidait sur un pic élevé perdu dans les brumes, son logis étant encastré dans la roche, donne un parallèle à l'image de la pagode suspendue dans l'espace $(k \bar{u} c h \bar{u})$.

Mais nous objectera-t-on, est-il légitime de déchiffrer une allusion aussi précise et profonde derrière une expression somme toute anodine? N'est-ce pas exagérer injustement la charge bouddhique de ces poèmes et ne nous laisserions-nous pas aller à une lecture arbitraire, ce que les Japonais appelleraient du yomi-komi? Je pourrais d'abord invoquer, pour défendre mon interprétation, le texte lui-même : l'échange de poèmes se déroule, nous l'avons remarqué, sur fond de Samâdhi du Lotus, hokke-sanmai, et il suffirait de renvoyer au savant ouvrage de Mme Kuo pour rappeler que cette 
pratique, selon les paroles mêmes de Zhiyi, « permet aux adeptes du Grand Véhicule de voir le bodhisattva Samantabhadra, le stūpa dans lequel paraissent les deux buddha, Śâkyamuni et Prabhūtaratna, ainsi que les buddha des dix directions ${ }^{14}$. " L'apparition de la pagode est bien liée au rituel qu'entendaient les protagonistes dans le lointain, même si l'on peut penser qu'il est terminé lorsque les poèmes sont composés. Le saint reclus jouerait alors effectivement sur les circonstances pour élaborer ses fioritures poétiques. Mais il y a autre chose. Je dois avouer que, dès la première lecture du poème de l'ermite, je l'avais compris tout naturellement comme une allusion à l'ouverture de la pagode céleste et que j'avais été plutôt surpris de voir ce rapprochement passé sous silence par les quelques commentateurs que j'ai consultés. Il se trouvait simplement que, dans les poèmes à thèmes bouddhiques (shakkyōka) que j'avais pu lire, la mention de l'ouverture d'une porte, et précisément avec ce terme toboso qui figure dans le poème de l'ermite, était liée à la scène de la pagode. C'est avant tout le cas de la Centurie du Lotus (Ei-hyakushu waka, Ei-Hokke-hyakushu-waka) de Jien 慈円 (1155-1225), auquel je me suis plus particulièrement intéressé ces dernières années. Le mot toboso apparaît deux fois dans cette « centurie » quelque peu hors norme, puisqu'elle compte en fait cent quarante-quatre poèmes.

21 Tout d'abord, sous la citation scripturaire Ils se rendent au pied des arbres précieux (Lotus, p. 228) tirée du chapitre XI, Jien donne :

\begin{tabular}{|l|l|}
\hline $\begin{array}{l}\text { 木のもとや } \\
\text { konomoto ya }\end{array}$ & Au pied des arbres \\
\hline $\begin{array}{l}\text { たからのとぼそ } \\
\text { takara no toboso }\end{array}$ & tandis que s'est ouverte \\
\hline $\begin{array}{l}\text { あけがたに } \\
\text { akegata ni }\end{array}$ & la porte précieuse \\
\hline $\begin{array}{l}\text { 数かぎりなき } \\
\text { kazu kagiri naki }\end{array}$ & en nombre infini se voient \\
\hline $\begin{array}{l}\text { 光をぞみる } \\
\text { hikari wo zo miru }\end{array}$ & les rais de lumière ${ }^{15}$ \\
\hline
\end{tabular}

La radiance provient des bouddhas venus assister à l'ouverture de la pagode de MaintTrésor.

Le second poème est plus intéressant encore ; placés sous la citation scripturaire Que la pagode de l'Éveillé Maint-Trésor retourne à son état précédent (Lotus, p. 342), nous avons les vers :

\begin{tabular}{|l|l|}
\hline $\begin{array}{l}\text { おほ空に } \\
\text { o-zora ni }\end{array}$ & Le Saint qui ouvrit \\
\hline $\begin{array}{l}\text { ひらきしやどの } \\
\text { hirakishi/hi[bi]kishi yado no }\end{array}$ & les portes du gîte \\
\hline
\end{tabular}




\begin{tabular}{|l|l|}
\hline $\begin{array}{l}\text { 戸ぼそをば } \\
\text { toboso wo ba }\end{array}$ & au vaste ciel \\
\hline $\begin{array}{l}\text { あけし聖や } \\
\text { akeshi hijiri ya }\end{array}$ & retentissant \\
\hline $\begin{array}{l}\text { 又もさしけむ } \\
\text { mata mo sashikemu }\end{array}$ & les aura-t-il donc refermées ? ${ }^{16}$ \\
\hline
\end{tabular}

24 Ce chapitre XXII « La passation » (Zokurui-hon) est sans doute la fin réelle du Sūtra; la prédication du Bouddha terminée, la terre revient à son état originel et la Pagode rentre sous terre ; le poème reflète l'angoisse discrète de Jien à l'idée de la disparition du message du Lotus. On aura remarqué qu'outre les deux termes " porte » et " ouvrir », le poème mentionne le Saint, hijiri, c'est-à-dire le Bouddha, avec le mot même déjà utilisé pour désigner l'ermite par Murasaki Shikibu. En tant que lecture japonaise du mot shōja (sct. ârya) signifiant le bouddha, le terme hijiri peut s'appliquer aux deux registres; il est tout à fait possible que l'auteur du Genji monogatari ait joué sur ce double sens, l'image de l'ermite ouvrant sa porte lui inspirant celle du Bouddha ouvrant la pagode.

Nous avons vu, ou cru voir, qu'il y a un nombre significatif d'allusions au Sūtra du Lotus dans tout cet épisode, toujours sur le mode ironique et détaché plutôt que sur le ton de la piété. Cela ne suffit pas à transformer dame Murasaki en Louise Michel ; elle s'amuse, certes, à transformer la venue du Prince radieux en une apparition à rebours : c'est le mondain qui se révèle aux religieux en une manifestation du phénoménal, pourrait-on dire, kengon au lieu du kenjitsu de la dogmatique Tendai, car si l'on prend le mot hana dans le sens qu'il a d'ordinaire dans les poème bouddhiques, il signifie aussi bien le Sūtra du Lotus que la pensée éveillée, le Bouddha que la réalité ultime ; mais il n'y a nulle volonté ici de tourner la foi en ridicule. Le Prince lui-même a d'ailleurs un très bref moment conscience de son état peccamineux et aspire à plus haut: "Je voudrais demeurer en cet état " (kauyau naru sumai mo semahoshū) se dit-il auprès du Préfet monacal, après avoir considéré l'horreur de ses péchés (waga tsumi no hodo osoroshū) et la rétribution qu'il peut en attendre dans une autre existence (mashite nochi no yo no imijikaru beki). Mais il reprend très vite le fil de ses préoccupations coutumières.

Ainsi, Murasaki Shikibu reprend sur le mode plaisant mais non dérisoire le vocabulaire $\mathrm{du}$ bouddhisme. On a vu qu'une contemporaine, Izumi Shikibu, avait fait un poème poignant sur les mêmes ténèbres qui sont évoquées ici par le Prince pour taquiner une suivante. Mais il y a une autre femme de lettres de la même époque qu'il n'est pas inutile de mentionner. Il s'agit d'Akazome Emon 赤染衛門, dont il nous reste le recueil poétique personnel, l'Akazome Emon-sh $\bar{u}^{17}$. Nous renvoyons à l'article classique de M. Yamada Shōzen sur l'histoire des shakkyōka pour ce qui concerne la naissance de ce genre $^{18}$; il suffira de rappeler que la pratique d'écrire un waka sur chacun des vingthuit chapitres du Sūtra du Lotus s'est instituée du $\mathrm{X}^{\mathrm{e}}$ au XI ${ }^{\mathrm{e}}$ siècle et que les recueils personnels de Kintō et d'Akazome sont les plus anciens semble-t-il à contenir ces séries de poèmes scripturaires (hōmonka) complètes. Nous ne parlerons pas de Kintō, mais nous regarderons la série lotusienne d'Akazome. Le poème qu'elle donne pour le chapitre XII « Don des Dieux », confirme bien que c'est l'épisode de la petite dragonne qui est pour elle le centre du chapitre : 


\begin{tabular}{|l|l|}
\hline わたつみの & watatsumino \\
\hline みやをいでたる & miya wo idetaru \\
\hline 程もなく & hodo mo naku \\
\hline さはりのほかに & sawari no hoka ni \\
\hline なりにけるかな & narinikeru kana \\
\hline
\end{tabular}

Sitôt sortie de son palais du fond des mers, au-delà des obstacles, elle s'est retrouvée d'une autre poétesse, la première à avoir consacré toute une collection de waka au bouddhisme ; il s'agit de la princesse impériale Senshi 選子内親王 (964-1035) et de son Recueil sur la production de la pensée d'Éveil, composé en 1012. C'est l'exacte contemporaine de dame Murasaki, de même que son recueil se superpose presque exactement au Roman du Genji. Elle y donne trente poèmes sur chacun des vingt-huit chapitres du Sūtra du Lotus, ainsi que sur les deux «sūtras d'ouverture et de fermeture ${ }^{19}$ ». Or, l'on y trouve au poème $n^{\circ} 35$, sous l'en-tête du chapitre « La vision de la pagode de matières précieuses » et la citation scripturaire : «L'Éveillé çâkyamuni, de l'index droit, ouvrit la porte de la pagode faite des sept matières précieuses. Il en sortit un grand bruit ${ }^{20} . .$. ", le poème suivant composé par la princesse et prêtresse :

\begin{tabular}{|l|l|}
\hline $\begin{array}{l}\text { 玉の戸を } \\
\text { tama no to wo }\end{array}$ & La porte précieuse \\
\hline $\begin{array}{l}\text { ひらきし時に } \\
\text { hirakishi toki ni }\end{array}$ & quand je l'eus ouverte \\
\hline $\begin{array}{l}\text { あはずして } \\
\text { awazu shite }\end{array}$ & je ne le rencontrai point \\
\hline $\begin{array}{l}\text { 明けぬよにしも } \\
\text { akenu yo ni shi mo }\end{array}$ & Me faudra-t-il errer \\
\hline $\begin{array}{l}\text { まどふべしやは } \\
\text { madou beshi ya wa }\end{array}$ & en cette nuit sans aurore ? ${ }^{21}$ \\
\hline
\end{tabular}

Nous n'avons pas ici à faire l'exégèse du poème, remarquons simplement que s'y trouve aussi imbriqué le motif de la nuit, que l'on a vu tout à l'heure. Bien que l'on trouve la forme simple to, plutôt que toboso, pour "porte ", il est manifeste que le thème de 
l'ouverture de la porte était déjà présent dans les shakkyōka de l'époque de Murasaki Shikibu et que notre interprétation du poème tel qu'il se présente dans le Roman $d u$ Genji est cohérente avec l'usage contemporain et postérieur. Soulignons aussi le parallélisme contrasté de tama no to "porte de matière précieuse » de Senshi avec le matsu no toboso « porte de (simple) pin » de dame Murasaki.

Je pense avoir ainsi montré qu'une conscience plus claire des références bouddhiques possibles permet une meilleure compréhension de passages apparemment anodins de cette œuvre aux sens infinis qu'est le Roman du Genji.

\section{NOTES}

1. Je dois encore remercier Mme Terada de m'avoir fait parvenir, après la lecture de cette communication, l'article de Mme Kubukihara Rei sur le Roman du Genji et le Sūtra du Lotus ( (Genji-monogatari to Hokekyō -Rokujō-miyasudokoro no tsumi e ») in Genji monogatari uta to jusei, Wakakusa shobō, 1997, pp. 315-324.

2. Pour reprendre la traduction de René Sieffert, à laquelle j'aurai recours lorsque je ne traduirai pas moi-même.

3. Sieffert, Le Dit du Genji, tome 1, POF, 1978, p. 164.

4. Amour, colère, couleur, Collège de France, 2000, p. 211.

5. Genji monogatari, tome 1, coll. « Nihon koten bungaku taikei » (NКBT), vol. 16, p. 192, n.9.

6. Cf. notre traduction du Lotus, p. 169 (Sūtra du Lotus, Fayard, 1997).

7. On l'appellera Dame Akashi (Akashi no ue). Son chemin croisera celui du Genji, qui assurera son pouvoir grâce à la princesse Akashi, fruit de leur liaison, laquelle deviendra impératrice.

8. 深入禪定了達諸法; Lotus, p. 240.

9. Je remercie Mme Terada de m'avoir soufflé cette suggestion fructueuse.

10. Lotus, p. 90.

11. Confession et contrition dans le bouddhisme chinois $d u V^{e}$ au $X^{e}$ siècle, EFEO, Paris, 1994, p. 87 et suiv.

12. Lotus, p. 221 et suivantes. Je modifie ici légèrement le texte afin de le mettre au plus serré du propos.

13. Amour, colère, couleur, op. cit., pp. 204 et suiv.

14. Confession et contrition, p. 89-90 (je simplifie un peu la typographie et mets les italiques), avec renvoi note 73 au chapitre XI du Sūtra du Lotus.

15. Shūgyoku shū, Shinpen kokka taikan, tome 3, Kadokawa shoten, 1985, p. 691 (poème $\mathrm{n}^{\circ} 2470$ ).

16. Ibid., p. 692 (poème $n^{\circ}$ 2515) ; il existe les deux versions hirakishi « qu'il a ouverte » et hibikishi «qui résonnèrent » pour le deuxième vers. Le verbe « résonner » fait écho, si l'on peut dire, à la suite du texte du Lotus, où il est dit : «Il en sortit un grand bruit, comme lorsque l'on ouvre les portes d'une ville en les déverrouillant» (p. 226). Le principe de la lectio difficilior voudrait que l'on choisisse hibiku, mais les meilleures éditions privilégient hiraku.

17. J'utilise ici l'édition de la série Shikashū zenshaku sōsho: Akazome Emon-shū zenshaku, Kazama shobō, Tōkyō, 1986. Les vingt-huit poèmes du Lotus se trouvent p. 377-409.

18. Yamada Shōzen, "L'origine et le développement des poèmes à thème bouddhique " dans Bukkyō bungaku kōza, vol. 4, Bensei-sha, Tōkyō, 1995, pp. 37-75. 
19. Voir la traduction anglaise abondamment commentée d'Edward Kamens: The Buddhist Poetry of the Great Kamo Priestess: Daisaiin Senshi and Hosshin Wakashū, Ann Arbor, Center for Japanese Studies, The University of Michigan, 1990.

20. Lotus, p. 226.

21. Hosshin waka shū, coll. «Shinpen kokka taikan », op. cit., p. 293 (poème nº 35).

\section{RÉSUMÉS}

La compréhension des nombreuses allusions au bouddhisme (particulièrement au Sūtra du Lotus) présentes dans le Genji permet de mieux appréhender l'œuvre de Murasaki Shikibu.

Understanding the references to Buddhism (especially the Lotus Sutra) in the Genji can lead to a better understanding of this work.

\section{INDEX}

Mots-clés : bouddhisme, Dit du Genji, Murasaki Shikibu (v. 973-v. 1014 ou 1025), Genji monogatari, méditation - bouddhisme, porte -- ouverture, Sūtra du lotus (Saddharma puṇdarīka), symbolisme bouddhique, Wakamurasaki キーワード : bukkyō 仏教, Genji monogatari 源氏物語, Murasaki Shikibu 紫式部 (v. 973-v. 1014 ou 1025), inja 隠者, zenjō 禅定, to (toboso) wo hiraku戸（枢）を開く, hokekyō 法華経, Wakamurasaki 若紫, bukkyō no shinborizumu 仏教のシンボリズム, Heian jidai 平安時代 (794-1185), shūkyōgaku 宗教学

Thèmes : sciences des religions Index chronologique : Heian

Keywords : Buddhist Art and Symbolism, Door Opening, Genji monogatari, Hermit, Meditation Buddhism, Saddharma puṇạarīka, Buddhism, Wakamurasaki 